\title{
Angiomiolipomas renales: presentación, tratamiento y resultado de 20 casos
}

\author{
Bestard Vallejo JE, Trilla Herrera E, Celma Domenech A, Pérez Lafuente M*, \\ de Torres Ramírez I**, Morote Robles J.
}

\author{
Servicio de Urología, *Angio-Radiología y **Anatomía Patológica del \\ Hospital General Universitario Vall d'Hebron. UAB. Barcelona.
}

Actas Urol Esp. 2008;32(3):307-315

\begin{abstract}
RESUMEN
ANGIOMIOLIPOMAS RENALES: PRESENTACIÓN, TRATAMIENTO Y RESULTADO DE 20 CASOS

Introducción: Los angiomiolipomas renales son tumores benignos. Ocasionalmente la ruptura espontánea de los mismos puede poner en peligro la vida de los pacientes. Tanto el tratamiento quirúrgico como la embolización arterial selectiva son tratamientos válidos para dichas lesiones.

Objetivos: Analizar los casos de angiomiolipomas renales tratados en nuestro centro, la presentación clínica y el tratamiento indicados en cada uno.

Material y método: Se revisan de forma retrospectiva 20 pacientes con angiomiolipomas renales tratados durante el período comprendido entre marzo de 1996 y marzo de 2006. Se describen las características de dichos tumores, el tratamiento recibido y los resultados obtenidos.

Resultados: Los pacientes afectados de esclerosis tuberosa (20\%) presentaban tumores múltiples y bilaterales, aunque de tamaño similar a los del resto de pacientes. El diagnóstico de síndrome de esclerosis tuberosa fue el único factor asociado a la ruptura espontánea de las lesiones. Cuatro de los 9 pacientes embolizados de urgencia presentaron resangrado (44,4\%) y 4 del total de los 16 casos embolizados (25\%) sufrieron el síndrome post-embolización, sin hallar ningún factor predictor para ninguna de las dos situaciones. Un 58,4\% de los tumores embolizados presentaron reducciones del tamaño inferiores a un tercio del inicial, caracterizándose éstos por ser múltiples, bilaterales y de mayor tamaño que el resto. No se hallaron diferencias significativas en los niveles de creatinina plasmática antes y después de cada tratamiento.

Conclusiones: Los angiomiolipomas renales parecen comportarse de forma más agresiva en los pacientes con síndrome de esclerosis tuberosa. No hemos hallado factores predictores de resangrado ni del síndrome post-embolización. Tanto la cirugía como la embolización arterial no parecen comprometer la función renal de los pacientes tratados.
\end{abstract}

Palabras clave: Angiomiolipoma renal. Embolización selectiva. Síndrome de esclerosis tuberosa

\section{ABSTRACT}

RENAL ANGIOMYOLIPOMA: PRESENTATION, TREATMENT AND RESULTS OF 20 CASES

Introduction: Renal angiomyolipoma is a benign tumour, but its spontaneous rupture may become threatening for patient's live. Both surgery and selective arterial embolization are accepted treatments for this lesion.

Objectives: Analyze renal angyolipoma treated in our center, their clinical outcome and treatment purposed in each case.

Material and Methods: We retrospectively analyse 20 cases of patients with renal angiomyolipoma treated in our centre from March 1996 to March 2006. We describe tumour characteristics, treatment followed and results obtained.

Results: Patients suffering from tuberous sclerosis $(20 \%)$ showed multiple bilateral tumours, with size similar to the rest of patients. Diagnosis of tuberous sclerosis was the only factor attached to spontaneous rupture of those lesions. Four of 9 emergency embolized patients (44.4\%) required from second treatment because of recurrent haemorrhage, and 4 of the 16 embolization episodes $(25 \%)$ presented post-embolization syndrome, both with no predicting factors attached. Reduction of less than one third of the inicial diameter was observed in $58.4 \%$ of embolized tumours, which used to be multiple, bilateral and of size larger to the rest. No significative differences were observed in plasmatic creatinine after and before treatments.

Conclusions: Renal angiomyolipoma may behave in an aggressive way in patients with tuberous sclerosis. No predicting factors of recurrent haemorrhage or post-embolization syndrome were observed. Both surgery and arterial embolization have proved not to compromise renal function in treated patients.

Keywords: Renal angiomyolipoma. Selective embolization. Tuberous sclerosis complex.

Abreviaturas: Angiomiolipoma (AML). Síndrome de esclerosis tuberosa (SET). Embolización arterial selectiva (EAS). Inestabilidad hemodinámica (IH). 
$\mathrm{L}$ os angiomiolipomas renales (AML) son tumores generalmente benignos formados por tejido graso maduro, vasos sanguíneos aberrantes y células musculares lisas ${ }^{1}$. Sus características histológicas fueron inicialmente descritas por Fischer ${ }^{2}$ en 1911, en un artículo en el que hacía referencia a ciertos tumores renales en pacientes con sindrome de esclerosis tuberosa (SET).

Si bien cada vez se tiende más a diagnosticar dichas lesiones a través de hallazgos en las pruebas de imagen, la ruptura espontánea sigue siendo una presentación clínica frecuente y potencialmente letal $^{3}$.

El tratamiento había sido eminentemente quirúrgico hasta que Moorhead et $\mathrm{al}^{4}$ en 1977 describieron el primer episodio de embolización arterial selectiva (EAS). Desde entonces ambas técnicas han demostrado buenos resultados a largo plazo, si bien la literatura describe cada vez más series de AML tratados mediante EAS.

El objetivo de nuestro estudio ha sido intentar identificar los factores que pueden determinar una ruptura espontánea del AML, los que predicen un resangrado posterior al tratamiento mediante EAS y los que predicen una reducción significativa de tamaño tras la embolización. También analizamos las características del AML en los pacientes con SET, comparándolos con las del resto de casos.

\section{MATERIAL Y MÉTODOS}

Hemos revisado los archivos informáticos de nuestro centro, identificando aquellos pacientes con AML tratados durante el periodo comprendido entre marzo de 1996 hasta marzo 2006. Se han tratado un total de 20 pacientes, de los cuales 8 (40\%) fueron tratados inicialmente mediante cirugía y 12 (60\%) mediante EAS.

En cada paciente identificamos las variables: forma de tratamiento (urgente/electivo), los niveles de creatinina plasmática (Crp en $\mathrm{mg} / \mathrm{dL}$ ), tensión arterial y nivel de hematocrito previo. La presencia de hipotensión arterial mantenida (TA sistólica $<90$ mmHg) la caída de 4 o más puntos de hematocrito en 24 horas o un hematocrito inicial por debajo de $25 \%$ se consideraron criterios de inestabilidad hemodinámica (IH). Las características de las lesiones en las pruebas de imagen (ECO, TAC y/o RNM), las características angiográficas en el caso de embolización y la técnica seguida y características anato- mo-patológicas en el caso de abordaje quirúrgico fueron igualmente evaluadas. En los casos de embolización de varias lesiones se tomó como referencia aquella de mayor tamaño.

Las complicaciones quirúrgicas y de las EAS han sido evaluadas por separado. El síndrome postembolización (SPE) se ha definido como el cuadro consistente en dolor lumbar, fiebre y reacción leucocitaria dentro de las 72 horas posteriores a la embolización. El seguimiento de los pacientes sometidos a EAS se ha evaluado mediante la reducción de las lesiones en las pruebas de imagen.

Las variables cualitativas de los pacientes se expresaron mediante su proporción en tanto por ciento (\%) y las variables cuantitativas mediante su media y desviación estándar. Para comparar las proporciones se utilizó el test de Fisher cuando se trataba de proporciones y el test de la U de Mann Whitney cuando se trataba de variables cuantitativas. Se tomaron como estadísticamente significativos valores de $\mathrm{p}<0,05$. Los resultados estadísticos han sido analizados mediante el paquete informático SPSS versión 12,0.

\section{RESULTADOS}

La edad media de los pacientes fue de 45,2 años (18-67), siendo el 75\% mujeres. En 14 pacientes existió algún tipo de clínica (70\%), mientras que 6 (30\%) fueron diagnosticados de forma incidental mediante pruebas de imagen. El dolor lumbar fue el síntoma más frecuente, presentándose en 11 pacientes $(78,5 \%)$, seguido del shock o cuadro vagal en 6 pacientes $(42,8 \%)$. Sólo se presentó hematuria macroscópica en un paciente $(7,1 \%)$.

Una paciente estaba afecta de linfangioleiomiomatosis pulmonar y 4 pacientes más (20\%) estaban afectados de SET. Las características de estos pacientes se resumen en la Tabla 1 .

Doce de los pacientes fueron tratados en un primer momento de forma urgente por ruptura espontánea (60\%), mientras que el resto fueron tratados de forma electiva (40\%), las características de unos y otros se resumen en la Tabla 2. Los valores analíticos, hemodinámicos y los hallazgos en las pruebas de imagen de los pacientes que requirieron de tratamiento urgente se exponen en la Tabla 3.

La TC fue la prueba de imagen más utilizada para confirmar el diagnóstico (75\%), seguida de la RNM (15\%). Tres de los pacientes se orientaron ini- 
Tabla 1. Características de los pacientes afectados de SET respecto de los no afectados.

\begin{tabular}{lccc}
\hline & SET* & NO SET & p \\
\hline Edad (años) & $45,7(\mathrm{DE} 16,2)$ & $45(\mathrm{DE} 15,4)$ & $>0,05$ \\
Pacientes varones n (\%) & $2(50 \%)$ & $3(18,75 \%)$ & $>0,05$ \\
Multiplicidad n (\%) & $4(100 \%)$ & $4(25 \%)$ & $>0,05$ \\
Bilateralidad n (\%) & $4(100 \%)$ & $2(12,5 \%)$ & $>0,05$ \\
Tamaño medio (cm) & $8,7(\mathrm{DE} 3,7)$ & $7,9(\mathrm{DE} 3,8)$ & $>0,05$ \\
Ruptura espontánea n (\%) & $4(100 \%)$ & $8(50 \%)$ & $>0,05$ \\
IH n (\%) & $4(100 \%)$ & $4(25 \%)$ & $>0,05$ \\
Hematoma en TC n (\%) & $4(100 \%)$ & $6(37,5 \%)$ & $>0,05$ \\
Sangrado activo en TC n (\%) & $4(100 \%)$ & $1(6.25 \%)$ & $>0,05$ \\
Total de pacientes n (\%) & $4(20 \%)$ & $16(80 \%)$ & \\
\hline
\end{tabular}

* SET $=$ Síndrome de esclerosis tuberosa.

Tabla 2. Características de los pacientes tratados en un primer momento de forma urgente respecto de los tratados de forma electiva

\begin{tabular}{lccc}
\hline & $\begin{array}{c}\text { Tratamientos } \\
\text { Urgentes }\end{array}$ & $\begin{array}{c}\text { Tratamientos } \\
\text { Electivos }\end{array}$ & $\mathbf{p}$ \\
\hline Varones n (\%) & $4(33,3 \%)$ & $1(12,5 \%)$ & $>0,05$ \\
Edad (años) & $45,6(\mathrm{DE} 14,8)$ & $44,5(\mathrm{DE} 16,6)$ & $>0,05$ \\
SET n (\%) & $4(33,3 \%)$ & $0(0 \%)$ & 0,02 \\
Multiplicidad n (\%) & $5(41,6 \%)$ & $3(37,5 \%)$ & $>0,05$ \\
Tamaño al debut (cm) & $8,8(\mathrm{DE} 3,1)$ & $7(\mathrm{DE} 4,5)$ & $>0,05$ \\
AML > 4 cm n (\%) & $10(83,3 \%)$ & $5(62,5 \%)$ & $>0,05$ \\
Aneurismas* n (\%) & $2(16,6 \%)$ & $0(0 \%)$ & $>0,05$ \\
Total de tratamientos n (\%) & $12(60 \%)$ & $8(40 \%)$ & \\
\hline
\end{tabular}

*Objetivado en los pacientes sometidos a angiografía (4 de los electivos y 9 de los urgentes). tiples (40\%), y en 5 pacientes eran bilaterales (25\%). Una paciente fue catalogada inicialmente como portadora de AML múltiple en un solo riñón que fue tratado mediante cirugía, si bien los estudios siguientes demostraron la aparición de nuevos AML en el riñón contralateral.

Se practicaron un total de 11 intervenciones quirúrgicas. Seis de ellas se realizaron con carácter urgente $(54,5 \%), 3$ como tratamiento de primera intención y 3 por persistencia de sangrado tras la EAS. En 5 pacientes se realizó nefrectomía parcial y en 6 nefrectomía radical. En 7 casos el abordaje se realizó por lumbotomía $(63,6 \%)$, en 3 laparotomía media $(27,2 \%)$ y en un caso se realizó nefrectomía parcial por vía laparoscópica (9\%).

Se detectó un incremento medio de Crp después de la intervención de $0,12 \mathrm{mg} / \mathrm{dL}(-0,30-0,53)$ que no alcanzó valor significativo. Sólo 1 paciente presentó unos niveles de Crp superiores a $2 \mathrm{mg} / \mathrm{dL}$ al año de la cirugía.
Tabla 3. Características de los pacientes que requirieron de tratamiento urgente.

\begin{tabular}{lcc}
\hline & n & \% \\
\hline Hematocrito $<25 \%$ & 4 & $33,3 \%$ \\
Creatinina $>2 \mathrm{mg} / \mathrm{dL}$ & 2 & $16,6 \%$ \\
Leuc $>15000 \times 10^{9} / \mathrm{L}$ & 2 & $16,6 \%$ \\
Inestabilidad hemodinámica & 10 & $83,3 \%$ \\
Transfusión en urgencias. & 7 & $58,3 \%$ \\
Hematoma en TC. & 10 & $83,3 \%$ \\
Sangrado activo en TC* & 5 & $45,45 \%$ \\
Edad (años) & 45,6 & - \\
\hline
\end{tabular}

*Objetivado en 5 de los 11 a los que se les administró contraste

cialmente como tumoraciones malignas (15\%), confirmándose con el estudio anatomopatológico el diagnóstico de AML (Fig. 1).

El diámetro medio de los AML fue de $8,75 \mathrm{~cm}$. (215.5). Ocho de los pacientes presentaban AML múl-
Un paciente tratado con nefrectomía parcial presentó una fístula urinaria que se resolvió mediante la colocación de un catéter ureteral. Otras complicaciones fueron un neumotórax, un episodio de neumonía y una pancreatitis, los dos últimos se resolvieron con tratamiento médico.

El examen microscópico de los especímenes quirúrgicos reveló 10 AML clásicos y un AML epitelioide (Fig. 2). Sólo uno de los pacientes intervenidos requirió de nuevo tratamiento por recidiva contralateral. El tiempo medio de seguimiento fue 80 meses (6-70).

Se realizó embolización en 12 pacientes como tratamiento de primera elección y en un caso al detectarse AML después de la cirugía. Se practicaron un total de 16 procedimientos endovasculares, requiriendo un paciente de 2 procedimientos por resangrado y otro de 3 procedimientos por persistencia de las lesiones. 


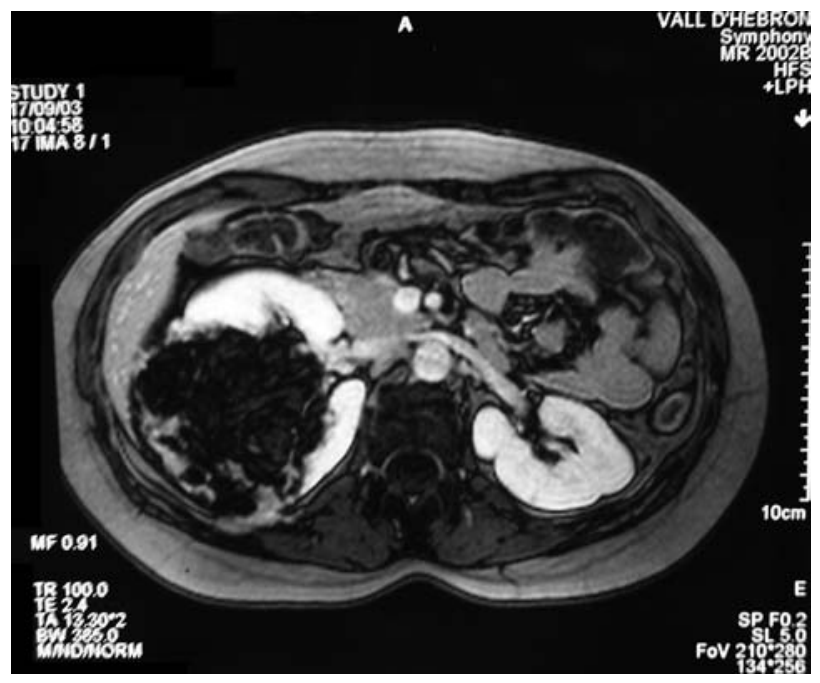

FIGURA 1. TC abdominal: Masa renal derecha de $7 \mathrm{~cm} \mathrm{de}$ diámetro, heterogénea y con múltiples áreas de densidad grasa, sugestiva de AML.

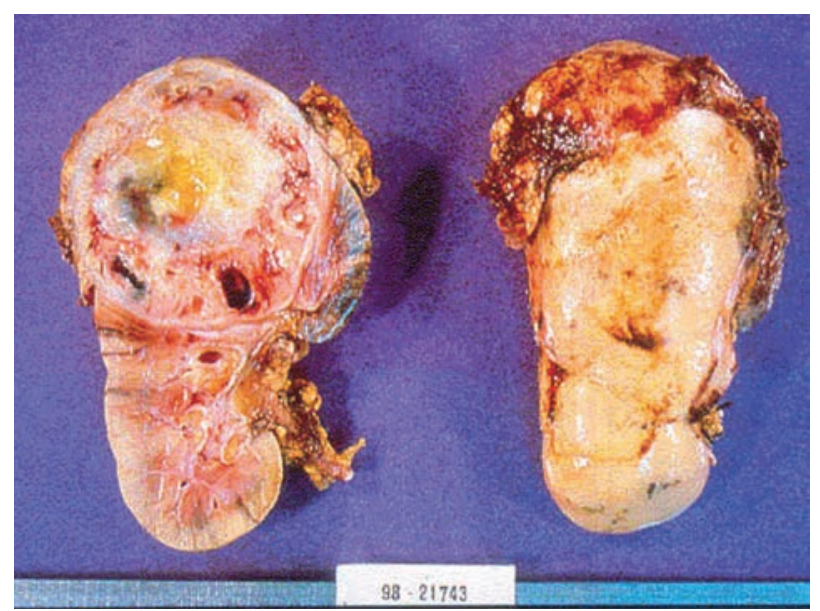

FIGURA 2. Tumoración renal bien delimitada con múltiples áreas grasas en su interior, diagnosticada como AML epitelioide.
Nueve de los 16 procedimientos $(62,5 \%)$ se realizaron de forma urgente por ruptura, requiriendo 4 de ellos $(44,4 \%)$ de un segundo tratamiento por persistencia del sangrado dentro de los primeros 30 días (3 cirugías y una reembolización). El tiempo medio entre el primer y el segundo tratamiento fue de 24.2 días (5-60). Las diferencias entre los pacientes de este grupo y el resto de pacientes embolizados de urgencias que no presentaron resangrado se resumen en la Tabla 4.

Se confirmó una avascularización adecuada mediante arteriografía posterior en 15 de las 16 embolizaciones (93,7\%) (Fig. 3 y Fig. 4).

Se detectaron complicaciones en 3 embolizaciones (18,7\%): 2 derrames pleurales y 2 cuadros sépticos sin focalidad en pacientes que no habían recibido cobertura antibiótica. El SPE se observó en 4 de

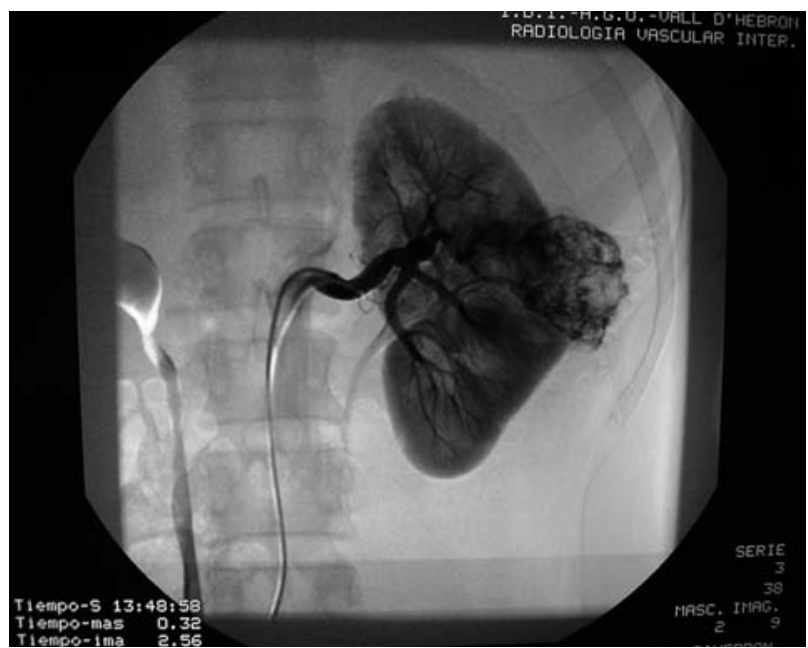

FIGURA 3. Arteriografia: AML de $4 \mathrm{~cm}$ en tercio medio del riñón izquierdo, apreciándose el importante componente vascular del mismo.

Tabla 4. Características de los pacientes embolizados de urgencias que presentaron resangrado respecto de los que no lo presentaron.

\begin{tabular}{lccc}
\hline & Resangrando & No resangrando \\
& n (\%) & n (\%) & $>0,05$ \\
\hline Edad & $40,5(\mathrm{DE} 16,8)$ & $51,4(\mathrm{DE} 11,7)$ & $>0,05$ \\
SET n (\%) & $2(50 \%)$ & $1(20 \%)$ & $>0,05$ \\
IH en el debut n (\%) & $4(100 \%)$ & $3(60 \%)$ & $>0,05$ \\
Múltiples n (\%) & $2(50 \%)$ & $2(40 \%)$ & $>0,05$ \\
Tamaño (cm) & $7,5(\mathrm{DE} 2,6)$ & $10(\mathrm{DE} 3,9)$ & $>0,05$ \\
Sangrado activo TC n (\%) & $2(50 \%)$ & $2(40 \%)$ & $>0,05$ \\
Sangrado activo en angiografía n (\%) & $1(25 \%)$ & $2(40 \%)$ & $>0,05$ \\
Aneurismas n (\%) & $1(25 \%)$ & $1(20 \%)$ & $5(55,5 \%)$ \\
Total de pacientes n (\%) & $4(44,4 \%)$ & \\
\hline
\end{tabular}




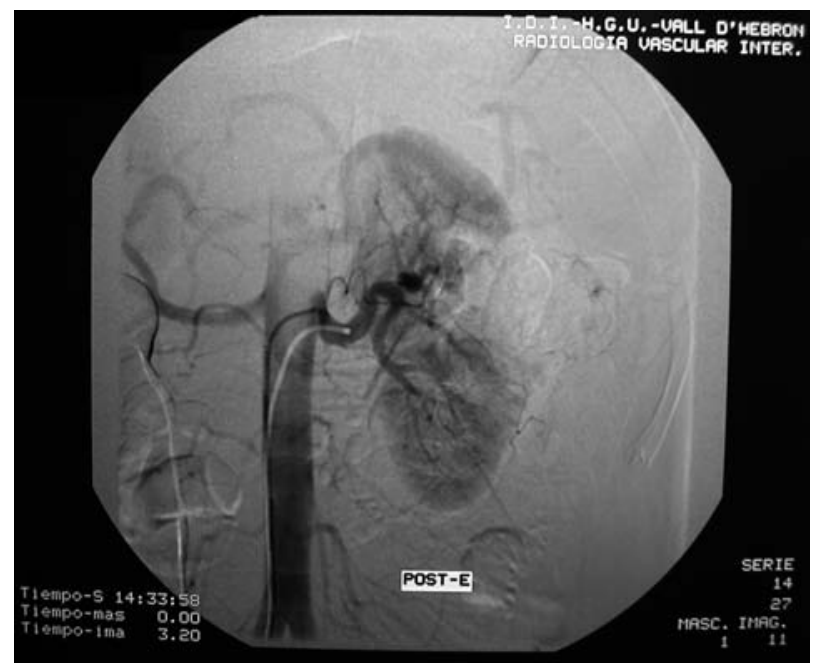

FIGURA 4. Arteriografía post-embolización. Se aprecia claramente la correcta avascularización respecto la imagen previa.

los 16 tratamientos (25\%), 2 en la misma paciente. En la Tabla 5 se resumen las características de este grupo de pacientes.

La diferencia de niveles de Crp entre antes y después de la EAS fue de -0,26 mg/dL (-0,58-0,68 $\mathrm{mg} / \mathrm{dL})$, sin alcanzar valor estadísticamente significativo. Ningún paciente presentó niveles de Crp superiores a $2 \mathrm{mg} / \mathrm{dL}$ al año de la EAS.

Se pudo efectuar el seguimiento en 12 de los episodios de EAS ( 1 paciente se perdió para el seguimiento, 1 se tuvo que reembolizar por resangrado y a otros 2 se les extirpó completamente el tumor durante la cirugía posterior). La técnica de imagen para realizar los controles fue en su mayor parte la TC $(76,9 \%)$, en 3 casos la RNM $(15,3 \%)$ y en uno la ecografía $(7,6 \%)$.
La evolución respecto al tamaño de las lesiones se resume en la Tabla 6.

El tiempo medio hasta que se objetivó una primera reducción del tamaño fue 6 meses (1,26-13,5 meses), y hasta la reducción máxima de 10,5 meses (3-20). Ninguno de los pacientes presentó aumento del tamaño tras alcanzar la reducción máxima, durante un seguimiento medio de 48 meses (12-117).

Tabla 6. Evolución del tamaño de los AML embolizados.

\begin{tabular}{lcc}
\hline & n & $\%$ \\
\hline Aumento & 2 & 16,6 \\
Estable & 3 & 25 \\
Reducción $<1 / 3$ & 2 & 16,6 \\
Reducción $>1 / 3$ & 5 & 41,6 \\
Total & 12 & $100 \%$ \\
\hline
\end{tabular}

En la Tabla 7 se resumen las características de los pacientes sometidos a EAS en función de la evolución del tamaño. Los 7 AML que no presentaron reducciones de su tamaño superiores a 1/3 eran mayores o iguales a $8 \mathrm{~cm}$.

El tiempo medio de seguimiento de los pacientes independientemente del tratamiento recibido fue de 61,75 meses (3-270). Un paciente se perdió para el seguimiento y otro sometido a nefrectomía falleció por un cuadro séptico en el contexto de hemodiálisis por insuficiencia renal de causa multifactorial.

Todos los demás pacientes se encontraban con buen estado general y función renal conservada. Hasta el momento ninguno de ellos ha vuelto a presentar recidiva clínica.
Tabla 5. Características de los pacientes que presentaron SPE respecto de los que no lo presentaron.

\begin{tabular}{lccc}
\hline & SPE$^{*}$ & NO SPE & p \\
\hline Edad (años) & $38,2(\mathrm{DE} 10,6)$ & $42,8(\mathrm{DE} 13,8)$ & $>0,05$ \\
Varones n (\%) & $0(0 \%)$ & $3(25 \%)$ & $>0,05$ \\
SET n (\%) & $0(0 \%)$ & $4(33,3 \%)$ & $>0,05$ \\
Electivos n (\%) & $3(75 \%)$ & $3(25 \%)$ & $>0,05$ \\
Tamaño (cm) & $8,6(\mathrm{DE} 1,8)$ & $9,2(\mathrm{DE} 4,1)$ & $>0,05$ \\
Hematomas en TC n (\%) & $1(25 \%)$ & $7(58,3 \%)$ & $>0,05$ \\
Embolización múltiple n (\%) & $1(25 \%)$ & $2(16,6 \%)$ & $>0,05$ \\
Sangrado en arteriografía n (\%) & $1(25 \%)$ & $2(16,6 \%)$ & $>0,05$ \\
Aneurismas n (\%) & $0(0 \%)$ & $2(16,6 \%)$ & $>0,05$ \\
Total de embolizaciones n (\%) & $4(25 \%)$ & $12(75 \%)$ &
\end{tabular}

*SPE $=$ Síndrome post-embolización.

\section{DISCUSIÓN}

Los AML representan actualmente entre el 1 y el $3 \%$ de todos los tumores renales. Son relativamente raros $\mathrm{y}$ suelen presentarse con mayor frecuencia en mujeres de mediana $\operatorname{edad}^{3}$.

En un estudio japonés en el que se practicaron pruebas de imagen renales a 17.941 voluntarios sanos se objetivaron imágenes sugestivas de AML en 24 $(0,13 \%)^{5}$. 
Tabla 7. Características de los AML que redujeron su tamaño en más de $1 / 3$ del diámetro inicial $(\mathrm{R}>1 / 3)$ respecto de los que no lo hicieron (Otros)

\begin{tabular}{lccc}
\hline & $\mathbf{R}>\mathbf{1 / 3}$ & Otros & $\mathbf{p}$ \\
\hline Edad (años) & $43,8(\mathrm{DE} 16,9)$ & $38,1(\mathrm{DE} 9,3)$ & $>0,05$ \\
SET n (\%) & $0(0 \%)$ & $2(28,5 \%)$ & $>0,05$ \\
Urgencia n (\%) & $3(60 \%)$ & $3(42,8 \%)$ & $>0,05$ \\
Múltiples n (\%) & $2(40 \%)$ & $6(85,7 \%)$ & $>0,05$ \\
Bilateral n (\%) & $0(0 \%)$ & $6(85,7 \%)$ & $>0,05$ \\
Embolización múltiple n (\%) & $0(0 \%)$ & $4(57,1 \%)$ & $>0,05$ \\
Tamaño cm & $7,4(\mathrm{DE} 2.8)$ & $10,7(\mathrm{DE} 3.7)$ & $>0,05$ \\
SPE n (\%) & $1(20 \%)$ & $1(14 \%)$ & $>0,05$ \\
Comprobación n (\%) & $4(100 \%)$ & $6(87,5 \%)$ & $>0,05$ \\
Total n (\%) & $5(41,6 \%)$ & $7(58,3 \%)$ & $>0,05$ \\
\hline
\end{tabular}

La relación entre AML y el SET siempre ha sido conocida. Entre el 50 y el $80 \%$ de los pacientes afectos por esta enfermedad presentan AML, siendo el sangrado de estos tumores la primera causa de muerte en los adultos de este grupo. A la inversa, un $20 \%$ de los pacientes con AML son portadores de dicho síndrome ${ }^{6}$.

En este grupo de pacientes los AML se presentan con igual frecuencia en ambos sexos, son de mayor tamaño que en los pacientes sin SET y con mayor frecuencia son múltiples y bilaterales.

En la Tabla 8 se detallan las características de los pacientes con AML en las series más amplias recientemente publicadas.

En el estudio microscópico de estos tumores se identifica tejido adiposo de aspecto normal, vasos tortuosos de paredes gruesas con poca elastina, y células musculares lisas. Englobados durante muchos años en el grupo de los hamartomas, actualmente se tiende a considerar los AML en un grupo aparte, el de los denominados PEComas o PEC tumours (tumores que presentan células epitelioides perivasculares), ya que ni la grasa ni la musculatura lisa formarian parte del riñón normal adulto. En nuestra serie una de las piezas resecadas resulto ser un AML epitelioide, una rara variedad que presenta un comportamiento incierto y puede debutar de forma agresiva, dando lugar a metástasis a distancia o diseminación local $^{1}$.
También parece que tienen una mayor tendencia al sangrado y a debutar clínicamente con cuadros de hemorragia masiva que los pacientes no afectados de SET $^{3}$.

Las series revisadas parecen confirmar estos datos, aunque el porcentaje de mujeres es superior al de varones en la mayoría de ellas ${ }^{6-8}$.

La serie de Ewalt et al. ${ }^{6}$ es la que describe una edad de presentación más temprana en los pacientes con SET, aspecto no confirmado en nuestro estudio.

En nuestra serie todos los pacientes con SET presentaban AML bilaterales y de tamaño similar al del grupo de pacientes sin SET, presentando todos ellos ruptura espontánea y requiriendo de tratamiento urgente.

La linfangioleiomiomatosis pulmonar es también rara y casi exclusiva del sexo femenino, presentándose de forma esporádica, asociada al SET y hasta

Tabla 8. Características de los pacientes con AML en las diferentes series. ${ }^{*}$ Hace referencia a la multipliciad o bilatertalidad de las embolizaciones. ${ }^{* * H a c e}$ referencia al porcentaje de AML con signos de sangrado en la TC.

\begin{tabular}{|c|c|c|c|c|c|c|c|c|c|}
\hline & $\mathbf{n}$ & $\begin{array}{c}\text { Edad } \\
\text { (años) }\end{array}$ & $\begin{array}{c}\text { Varones } \\
(\%)\end{array}$ & $\begin{array}{c}\text { Tamaño } \\
\text { (cm) }\end{array}$ & $\begin{array}{l}\text { SET } \\
(\%)\end{array}$ & $\begin{array}{c}\text { Múltiples } \\
\text { (\%) }\end{array}$ & $\begin{array}{c}\text { Bilaterales } \\
(\%)\end{array}$ & $\begin{array}{c}\text { Hallazgo } \\
\text { (\%) }\end{array}$ & $\begin{array}{c}\text { Tratamiento } \\
\text { urgente } \\
(\%)\end{array}$ \\
\hline Williams et al. 2006 & 16 & 29,7 & $25 \%$ & 10,6 & $100 \%$ & $?$ & $12,5 \%$ & $37,5 \%$ & $?$ \\
\hline Kothary et al. 2005 & 19 & 48,5 & $10,5 \%$ & $?$ & $52,6 \%$ & $52,6 \%$ & $\mathrm{a} ?$ & $15,7 \%$ & $36,8 \%$ \\
\hline Ewalt et al. 2005 & 16 & 23,1 & $37,5 \%$ & $4-21$ & $100 \%$ & $56,2 \% *$ & $6,25 \% *$ & $81,2 \%$ & $?$ \\
\hline Lee et al. 1997 & 15 & 34 & $20 \%$ & 8,6 & $40 \%$ & $?$ & $20 \%$ & $0 \%$ & $38 \% * *$ \\
\hline Mourikis et al. 1999 & 5 & 30 & $40 \%$ & 7 & $40 \%$ & $40 \%$ & $40 \%$ & $0 \%$ & $100 \%$ \\
\hline Yamakado et al. 2002 & 23 & 43 & $26 \%$ & 6,9 & $13 \%$ & $30,4 \%$ & $14,2 \%$ & $?$ & $34,7 \%$ \\
\hline Heinderich et al. 2001 & 28 & 55,6 & $14,2 \%$ & 5,5 & $3,5 \%$ & $7,14 \%$ & $7,14 \%$ & $?$ & $17,8 \%$ \\
\hline Fazeli et al. 1998 & 27 & 50 & $29 \%$ & 7,4 & $7,4 \%$ & $32 \%$ & $32 \%$ & $48 \%$ & $22,3 \%$ \\
\hline
\end{tabular}


en un 50\% de las pacientes a AML. Algún autor refiere que estas lesiones pulmonares son metástasis del AML renal ${ }^{9}$. La única paciente de nuestra serie con esta patología presentó un tumor único de $9,5 \mathrm{~cm}$ que se resolvió con EAS.

La utilización frecuente de pruebas de imagen ha favorecido el diagnóstico del AML y del resto de tumores renales en general (hasta el $81,2 \%$ de la serie de Ewalt et al) ${ }^{6}$. En nuestra serie se diagnosticaron como hallazgo incidental un total de 6 pacientes $(30 \%)$.

La presencia de calcificaciones intratumorales en la $\mathrm{TC}^{10}$ o de un crecimiento rápido, de más de 0,5 $\mathrm{cm}$. al año ${ }^{11}$ debe hacernos dudar del diagnóstico de benignidad. El porcentaje de lesiones que se intervienen con el diagnóstico inicial de malignidad varía entre el $33 \%$ y el $64 \%$ según las series ${ }^{12,13}$, si bien en la nuestra no supera el $15 \%$.

El tratamiento de los AML está indicado ante la presencia de clínica o el riesgo de complicaciones. En un estudio prospectivo de AML de diferentes tamaños, Oesterling et al. $^{14} \mathrm{y}$ posteriormente Steiner et al. ${ }^{15}$ observaron cómo aquellos de tamaño mayor a $4 \mathrm{~cm}$ tenían mayor predisposición al crecimiento y sangrado, recomendando su tratamiento de forma preventiva. Posteriormente, Dickinson et al. ${ }^{16}$ concluyen que, si bien la probabilidad de crecimiento y sangrado era patente para los tumores por encima de $8 \mathrm{~cm}$, no siempre era así para aquellos que presentaban diámetros intermedios entre 4 y 8 $\mathrm{cm}$. Yamakado et $\mathrm{al}^{17}$ en su serie recogen diferencias estadísticamente significativas entre el diámetro mayor de los AML que presentaron ruptura espontánea respecto los que no $(11,4 \mathrm{~cm}$ vs $5 \mathrm{~cm})$, siendo los primeros siempre mayores de $4 \mathrm{~cm}$.

Además del tamaño, Kothary et $\mathrm{al}^{8}$ determinan en una serie de 29 AML que la presencia de aneurismas superiores a $5 \mathrm{~mm}$ proporciona una especificidad superior para predecir el sangrado que el tamaño. Sin embargo, la presencia o no de aneurismas varía en diferentes series entre un $75 \%$ y un $5,2 \%$, requiriéndose la angiografía en la mayoría de casos para su detección. En sólo uno de los 22 pacientes de esta serie se pudo objetivar en la TC con contraste. No existen estudios que avalen la ecografía-doppler en su valoración ${ }^{7,17}$.

En nuestra serie ni la edad, sexo, multiplicidad o tamaño parece ser un criterio predictor de ruptura espontánea, siendo el diagnóstico de SET el único factor significativo. La incidencia de aneurismas fue baja (sólo se demostraron mediante angiografía en 2 pacientes y en ningún caso en la TC) y no fue indicativa de riesgo de ruptura espontánea.

En la Tabla 9 se recogen las características del tratamiento y seguimiento de los pacientes de las series anteriormente citadas.

La cirugía ha sido el tratamiento clásico de elección para estos tumores. Muchos autores defienden esta opción dada la improbable recidiva tras la exéresis completa y la falta de síndrome post-

Tabla 9. Tratamiento y seguimiento de los pacientes con AML en las diferentes series

\begin{tabular}{|c|c|c|c|c|c|c|c|c|c|}
\hline & $\mathbf{n}$ & Tratamiento & Material & Aneurismas & SPE & $\begin{array}{c}\text { Resangrado } \\
\text { precoz }\end{array}$ & $\begin{array}{l}\text { Pacientes } \\
\text { con } \\
\text { reducción }\end{array}$ & $\begin{array}{l}\text { Más de un } \\
\text { tratamiento }\end{array}$ & $\begin{array}{l}\text { Seguimiento } \\
\text { (meses) }\end{array}$ \\
\hline \multirow{2}{*}{ Williams et al. 2006} & 16 & EAS & CPV & & & & & & \\
\hline & & & Coils & $75 \%$ & $6,25 \%$ & $0 \%$ & $93,7 \%$ & $0 \%$ & 40 \\
\hline Kothary et al. 2005 & 19 & EAS & Etanol & $5,2 \%$ & $100 \%$ & $0 \%$ & $?$ & $31,6 \%$ & 51 \\
\hline Ewalt et al. 2005 & 16 & EAS & $\mathrm{CPV}$ & $12,5 \%$ & $68,7 \%$ & $0 \%$ & $100 \%$ & $0 \%$ & $?$ \\
\hline Lee et al. 1997 & 15 & EAS & Etanol & $6,6 \%$ & $80 \%$ & $13,3 \%$ & $60 \%$ & $13,3 \%$ & 35,6 \\
\hline \multirow[t]{2}{*}{ Mourikis et al. 1999} & 5 & EAS & $\mathrm{CPV}$ & & & & & & \\
\hline & & & Coils & $?$ & $40 \%$ & $20 \%$ & $?$ & $60 \%$ & 22.7 \\
\hline Yamakado et al. 2002 & 23 & EAS Cirugía & Etanol & $30,4 \%$ & $?$ & $0 \%$ & $66 \%$ & $0 \%$ & 45,9 \\
\hline Heidenreich et al. 2001 & 28 & Cirugía & , & , & , & , & , & $0 \%$ & 58 \\
\hline Fazeli et al. 1998 & 17 & Cirugía & , & , & , & , & , & $0 \%$ & 39 \\
\hline
\end{tabular}

$\mathrm{CPV}=$ Cloruro de polivinilo 
embolización ${ }^{13,18,19}$. Kennelly et al. ${ }^{20}$ defienden la exéresis de aquellos tumores de menor tamaño y la embolización de los que sean inabordables, mientras que otros como Nelson et al ${ }^{3}$ relegan la cirugía a aquellos pacientes con sospecha de malignidad o los que no responden a un primer tratamiento de EAS.

En los últimos años la nefrectomía parcial se ha estandarizado como el tratamiento de elección en muchos casos, aplicándose no sólo para tumores pequeños, sino incluso para tumores mayores como los descritos por Fazeli et $\mathrm{al}^{12}$. En su serie y en la de Heidenreich et al. ${ }^{13}$ se descarta que haya pérdida significativa de función renal tras la cirugía, tanto en los pacientes birrenos cómo monorrenos, así como que este empeoramiento tenga relación alguna con el tamaño tumoral. La fístula urinaria es la complicación más frecuente (alrededor de un 10\% en las series) seguidas de las infecciones. En nuestra serie tampoco observamos una pérdida significativa de función renal después de la cirugía, siendo las complicaciones similares a las descritas en la literatura.

El abordaje laparoscópico también se utiliza para el tratamiento electivo de estos tumores, si bien el tamaño que muchos AML alcanzan lo hace difícil $^{21}$.

La embolización estaría indicada en tumores múltiples, en pacientes con elevado riesgo quirúrgico y sobre todo en el tratamiento urgente de AML renales sangrantes. La tasa de complicaciones de este procedimiento se sitúa alrededor del 10\%, siendo las sépticas, especialmente el absceso renal, la más frecuente ${ }^{3}$. Otras complicaciones menos frecuentes son la migración del material de embolización, la licuefacción estéril y el derrame pleural reactivo ${ }^{6,22-24}$. En nuestra serie se observaron complicaciones hasta en un $18,7 \%$, siendo el derrame pleural y los problemas sépticos los más frecuentes.

No considerado como complicación según la mayoría de los autores, el síndrome post-embolización se presenta entre el 6 y el 100\% de los casos y no requiere más que de tratamiento sintomático ${ }^{3}$. En nuestra serie es un evento que se presentó en 4 de los 16 procedimientos (25\%), sin hallarse ningún factor predictor asociado.

Después de la EAS el resangrado se ha descrito hasta en un 20\% de los $\operatorname{casos}^{25}$. En nuestra serie este porcentaje es algo superior, sin que se haya detectado tampoco ningún factor predictor del mismo.
La mayoría de artículos también coinciden en que no existen variaciones significativas de la función renal después de embolizar ${ }^{7}$, como observamos en nuestra serie.

Ante la falta de datos anatomopatológicos el éxito de la EAS se relaciona con la reducción del tamaño de las lesiones. Si bien no en todas las series se cuantifica el grado de dicha reducción, Williams et al. ${ }^{7}$ observaron que la reducción media del volumen de los AML embolizados era de alrededor del 56\%, mientras que Yamakado et al ${ }^{17}$ detectaron sólo una reducción media del 26\%. En su serie se establece que el porcentaje de tumores que reducían el tamaño era similar para aquellos embolizados de forma urgente respecto a los electivos (57\% vs 60\%), si bien eran los segundos los que reducían su tamaño en mayor proporción ( $22 \%$ vs 33\%). Nuestra casuística presenta una tasa de reducciones del 58,2\%, siendo superior al 33\% del diámetro inicial sólo el $41,6 \%$. La proporción de pacientes que redujeron significativamente su tamaño fue similar en urgentes y electivos. Esta tasa de reducción es inferior a la mayoría de series $6,7,17,24$. El tamaño, la bilateralidad y la multiplicidad de las lesiones son factores que se ven con más frecuencia en los AML que no reducen su tamaño en más de un tercio, aunque sin alcanzar significación estadística. Tampoco la comprobación de una correcta avascularización fue predictor para dicha reducción de tamaño.

Según Han et al. ${ }^{23}$ y Lee et al. ${ }^{24}$ la primera disminución de tamaño no se produce hasta los 12 meses de la EAS. En nuestra serie el tiempo medio hasta que se objetivó una primera reducción del tamaño fue de 6 meses y hasta la máxima reducción de 10,5 meses.

En cuanto a las recurrencias, Kothary et $\mathrm{al}^{8}$ las definen como el incremento de $2 \mathrm{~cm}$ de diámetro en las pruebas de imagen, y describe una tasa del $31 \%$, la cual se produce en un tiempo medio de 78 meses. Todos los pacientes en los cuales se produjeron las recurrencias habían desarrollado SET y presentaban AML múltiples y bilaterales. La recurrencia no fue objetivada en ninguno de nuestros pacientes.

\section{CONCLUSIONES}

En nuestra experiencia los AML en pacientes con SET tienen una mayor tendencia a la ruptura espontánea independientemente de otros factores 
como el tamaño o la edad, y, por tanto, deberían ser sometidos a un seguimiento más cercano que el resto de pacientes.

Tanto la cirugía como la EAS ofrecen buenos resultados cuando se aplican de forma electiva, si bien el resangrado constituye una complicación relativamente frecuente en las embolizaciones por ruptura espontánea. Ni para este ni para el síndrome post-embolización hemos hallado factores predictores asociados.

La multiplicidad, bilateralidad y el mayor tamaño de las lesiones parece condicionar un mayor riesgo de no alcanzar una disminución satisfactoria de estas lesiones, aunque sin alcanzar significación estadística. Tampoco la comprobación de una correcta avascularización posterior a la EAS ha demostrado predecir esta reducción.

Por último, ni la embolización arterial ni el tratamiento quirúrgico han demostrado alteraciones significativas sobre la función renal.

\section{REFERENCIAS}

1. Hornick JL, Fletcher CD. PEComa: what do we know so far? Histopathology 2006; Jan 48(1):75-82.

2. Fischer W: Die Nierentumoren bei der Tuberosen Hirnsklerose. Beitr Pathol Anat 1911; 50: 235-282.

3. Nelson CP, Sanda MG: Contemporary diagnosis and management of renal angiomyolipoma. J Urol 2002 Oct;168(4 Pt 1): 1315-1325.

4. Moorhead JD, Fritzsche P, Hadley HL. Management of hemorrhage secondary to renal angiomyolipoma with selective arterial embolization. J Urol 1977 Jan;117(1):122-123.

5. Fujii Y, Ajima J, Oka K, Tosaka A, Takehara Y. Benign renal tumors detected among healthy adults by abdominal ultrasonography. Eur Urol. 1995;27(2):124-127.

6. Ewalt DH, Diamond N, Rees C, Sparagana SP, Delgado M, Batchelor L, Roach ES. Long-term outcome of transcatheter embolization of renal angiomyolipoma due to tuberous sclerosis complex. J Urol 2005;174(5):1764-1766.

7. Williams JM, Racadio JM, Johnson ND, Donnelly LF, Bissler JJ: Embolization of renal angiomyolipomata in patients with tuberous sclerosis complex. Am J Kidney Dis. 2006 Jan;47(1):95-102.

8. Kothary N, Soulen MC, Clark TW, Wein AJ, Shlansky-Goldberg $\mathrm{RD}$, Crino PB et al. Renal angiomyolipoma: long-term results after arterial embolization. J Vasc Interv Radiol. 2005 Jan; $16(1): 45-50$

9. Yu J, Astrindis A, Howard S, Henke EP: Estradiol and tamoxifen stimulates LAM-associated angiomyolipoma cell growth and activate both genomic and nongenomic signaling pathways. Am J Physiol Lung Cell Mol Physiol. 2004 Apr:286(4);694-700.

10. Hammadeh MY, Thomas K, Philp T, Singh M. Renal cell carcinoma containing fat mimicking angiomyolipoma: demonstration with CT scan and histopathology. Eur. Radiol 1998;8(2): 228-229.
11. Patel U, Simpson E, Kingswood JC, Saggar-Malik AK.Tuberose sclerosis complex: analysis of growth rates aids differentiation of renal cell carcinoma from atypical or minimal-fat-containing angiomyolipoma. Clin Radiol. 2005 Jun;60(6):665-673.

12. Fazeli-Matin S, Novick AC. Nephron-sparing surgery for renal angiomyolipoma. Urology. 1998 Oct;52(4):577-583.

13. Heidenreich A, Hegele A, Varga Z, von Knobloch R, Hofmann R. Nephron-sparing Eur Urol. 2002;41(3):267-273.

14. Oesterling JE, Fishman EK, Goldman SM, Marshall FF. The management of renal angiomyolipoma. J Urol. 1986 Jun;135 (6): 1121-1124.

15. Steiner MS, Goldman SM, Fishman EK, Marshall FF. The natural history of renal angiomyolipoma. J Urol. 1993 Dec;150(6): 1782-1786.

16. Dickinson M, Ruckle H, Beaghler M, Hadley HR. Renal angiomyolipoma: optimal treatment based on size and symptoms. Clin Nephrol. 1998 May;49(5):281-286.

17. Yamakado K, Tanaka N, Nakagawa T, Kobayashi S, Yanagawa M, Takeda K. Renal angiomyolipoma: relationships between tumor size, aneurysm formation, and rupture. Radiology 2002 Oct;225:78-82.

18. Elhence V, Mehta B, Jain M, Gupta RK. Renal angiomyolipoma: a clinico-pathological study or eleven cases. Indian $\mathrm{J}$ Cancer. 2002 Jun;39(2):55-60.

19. Marszalek M, Ponholzer A, Brossner C, Wachter J, Maier U, Madersbacher S. Elective open nephron-sparing surgery for renal masses: single-center experience with 129 consecutive patients. Urology 2004 Jul;64(1):38-42.

20. Kennelly MJ, Grossman HB, Cho KJ. Outcome analysis of 42 cases of renal angiomyolipoma. J Urol. 1994 Dec;152(6 Pt 1): 1998-91.

21. Steinberg AP, Kilciler M, Abreu SC, Ramani AP, Ng C, Desai MM et al. Laparoscopic nephron-sparing surgery for two or more ipsilateral renal tumors. Urology. 2004 Aug;64(2):255-228.

22. Hamlin JA, Smith DC, Tayor FC, McKinney JM, Ruckle HC, Hadley HR. Renal angiomyolipomas: long-term follow-up of embolization for acute hemorrhage. Can Assoc Radiol J. 1997 Jun;48(3): 191-198.

23. Han YM, Kim JK, Roh BS, Song HY, Lee JM, Lee YH et al. Renal angiomyolipoma: selective arterial embolization-effectiveness and changes in angiomyogenic components in long-term follow-up. Radiology. 1997 Jul;204(1):65-70.

24. Lee W, Kim TS, Chung JW, Han JK, Kim SH, Park JH. Renal Angiomyolipoma: Embolotherapy with mixture of Alcohol and Iodized Oil. J Vasc Intervent Radiol. 1998 Mar-Apr;9(2):255-261.

25. Mourikis D, Chatziioannou A, Antoniou A, Kehagias D, Gikas D, Vlahos L. Selective arterial embolization in the management of symptomatic renal angiomyolipomas. Eur J Radio. 1999 Dec;32:153-159.

Correspondencia autor: Dr. J.E. Bestard Vallejo Servicio de Urología.

Hospital General Universitario Vall d'Hebrón

Passeig de la Vall d'Hebrón, 119-129 - 08035 Barcelona

Tel.: 932746000

E-mail autor: 38625jbv@comb.es

Información artículo: Original - Patología renal benigna - Angiom.

Trabajo recibido: junio 2007

Trabajo aceptado: septiembre 2007 